\title{
Generation and Characterization of a Monoclonal Antibody Against prM Protein of West Nile Virus
}

\author{
Li-Ping Guo, Hong Huo, Xiao-Lei Wang, Zhi-Gao Bu, and Rong-Hong Hua
}

West Nile virus (WNV), which is an emerging pathogenic flavivirus with increasing distribution worldwide, is the cause of major human and animal health concerns. The pre-membrane (prM) protein of WNV is cleaved during maturation by the furin protease into the structural protein $\mathrm{M}$ and a pr-segment. In this study we generated and characterized a monoclonal antibody (MAb) against the WNV prM protein. Western blot analysis showed that the $\mathrm{MAb}$ reacted with WNV prM specifically. Immunohistochemistry assays demonstrated that the MAb recognized native prM protein in transfected BHK-21 cells. Preliminary studies were performed to identify the epitope recognized by the MAb using a set of synthesized overlapping peptides spanning the whole length of the prM protein. The MAb reported here may provide a valuable tool for the further exploration of the biological properties and functions of the prM protein and may also be developed for potential clinical applications.

\section{Introduction}

W EST Nile VIRUS (WNV) IS A ZOONOTIC mosquitotransmitted arbovirus belonging to the genus Flavivirus in the family Flaviviridae and is widely prevalent in Africa, Southern Europe, Russia, the Middle East, India, Australia, and North America. ${ }^{(1,2)}$ Since its first large outbreak in Romania in $1996,{ }^{(3)} \mathrm{WNV}$ has become a major public health and veterinary concern. In 2012, the US experienced a WNV outbreak, with 5387 cases of WNV disease reported, including 243 deaths. ${ }^{(4)}$

WNV was a single-stranded (positive sense) RNA virus with a genome of approximately $11 \mathrm{~kb}$ that encodes three structural proteins $(\mathrm{C}$, prM/M, and $\mathrm{E})$ and seven non-structural proteins (NS1, NS2A, NS2B, NS3, NS4A, NS4B, and NS5). ${ }^{(5,6)}$ The prM protein comprises 167 amino acids, with a molecular weight of 20 to $26 \mathrm{kDa}$. The prM protein, which acts as a chaperone that assists in the maturation of the E protein, interacts with $\mathrm{E}$ as a prM/E heterodimer, which is important for the formation of immature virions. ${ }^{(7,8)}$ The pr peptide is contained within the amino terminal region of the prM protein and is cleaved from prM by the protease furin in the trans-Golgi apparatus during release of the virus from the cell. The proteolytic cleavage of prM to generate the $\mathrm{M}$ protein results in the formation of a fusion competent particle. ${ }^{(9,10)}$ However, the functional role of prM in replication, virus infectivity, and induction of neutralizing activity ${ }^{(1)}$ is not fully understood.

In this study we generated a hybridoma cell line secreting a monoclonal antibody (MAb) specific for the pr region of the WNV prM protein. We subsequently characterized the MAb produced, which may be a useful tool for further investigations of the characteristics and functions of the prM protein, and as a potential candidate for clinical applications in the diagnosis and therapy of WNV.

\section{Materials and Methods}

\section{Cell lines, virus, and other reagents}

Baby hamster kidney (BHK-21) cells and SP2/0 myeloma cells were cultured in RPMI-1640 medium (Hyclone, Beijing, China) supplemented with $10 \%$ fetal calf serum (PAA, Somerset, United Kingdom) and antibiotics (100 IU/mL penicillin and $0.1 \mathrm{mg} / \mathrm{mL}$ streptomycin). All cells were maintained in a humidified $5 \% \mathrm{CO}_{2}$ atmosphere at $37^{\circ} \mathrm{C}$. Inactivated WNV (strain Chin-01) cell cultures and rabbit anti-WNV sera were kindly provided by Dr. Cheng-Feng Qin (Beijing Institute of Microbiology and Epidemiology, Beijing, China).

\section{Expression and purification of prM protein}

The recombinant plasmids were constructed and confirmed in our laboratory prior to transformation of E. coli ER2523 (NEB Express, Ipswich, MA) cells for protein expression. Recombinant protein was expressed according to a previously described procedure. ${ }^{(12)}$ Briefly, overnight cultures of the transformed cells were diluted 1:100 in 50 mL Luria-Bertani (LB) broth containing $100 \mu \mathrm{g} / \mathrm{mL}$ ampicillin at $37^{\circ} \mathrm{C}$. When the $\mathrm{OD}_{600}$ reached 0.6 , isopropyl-D-thiogalactopyranoside (IPTG; Sigma, St. Louis, MO) was added to the broth at a 
final concentration of $0.3 \mathrm{mM}$ to induce protein expression and the cells were incubated at $16^{\circ} \mathrm{C}$ overnight. Subsequently, bacterial cells were removed from the growth medium by centrifugation at $5000 \mathrm{~g}$ for $15 \mathrm{~min}$ and lysed by sonication in cold phosphate-buffered saline (PBS, pH 7.4). Predominant expression of the recombinant prM protein in the maltosebinding protein (MBP) fusion protein was confirmed by sodium dodecyl sulfate-polyacrylamide gel electrophoresis (SDS-PAGE, 12\%) and Western blotting. The fusion protein was affinity-purified for use as an immunogen with an amylose resin column.

\section{Generation, selection, and purification of monoclonal antibodies against prM}

The purified recombinant protein was used as an immunogen in mice. Hybridomas secreting anti-prM antibodies were generated according to a previously described method. ${ }^{(13)}$ Female BALB/c mice (aged 5 weeks) were immunized subcutaneously with recombinant protein emulsified with Freund's complete adjuvant (Sigma, St. Louis, MO). Two booster injections were given at 2-week intervals with the same immunogen emulsified in an equal volume of Freund's incomplete adjuvant (Sigma). A final intraperitoneal booster immunization of soluble prM protein without adjuvant was administered 3 days before cell fusion. Three days after the final administration, the mice were euthanized humanely; spleen cells were harvested and subsequently fused with SP2/ 0 myeloma cells (5:1 ratio) using polyethylene glycol (PEG 4000, Sigma). The hybridoma cells were seeded into 96-well plates and selected in HAT medium (RPMI-1640 medium containing $10 \%$ fetal bovine serum, $100 \mathrm{mg} / \mathrm{mL}$ streptomycin, $100 \mathrm{IU} / \mathrm{mL}$ penicillin, $100 \mathrm{mM}$ hypoxanthine, $16 \mathrm{mM}$ thymidine, and $400 \mathrm{mM}$ aminopterin). On days 3, 6, and 9, half of this medium was removed and replaced with fresh medium containing HAT. After HAT/HT medium selection, culture supernatants of surviving clones were screened for antibody reactivity and specificity by indirect enzyme-linked immunosorbent assay (ELISA). Positive clones were subcloned three times by the limiting dilution method. Selected clones were cultured in the peritoneal cavities of atolinprimed BALB/c mice to obtain ascitic fluid. MAb isotypes were determined with a mouse MAb isotyping kit according to the manufacturer's instructions (Hycult Biotechnology, Uden, The Netherlands).

\section{Enzyme-linked immunosorbent assay}

Ninety-six well microtiter plates were coated with purified fusion protein in $0.1 \mathrm{M}$ carbonate buffer $(\mathrm{pH} 9.6)$ at $4^{\circ} \mathrm{C}$ overnight and blocked with $5 \%$ skimmed milk for $2 \mathrm{~h}$ at $37^{\circ} \mathrm{C}$. After washing three times with PBST (PBS plus 0.5\% Tween20), $100 \mu \mathrm{L}$ of hybridoma supernatant containing MAbs were added to each well and incubated at $37^{\circ} \mathrm{C}$ for $1 \mathrm{~h}$, followed by three washes with PBST. The bound MAbs were detected with horseradish peroxidase (HRP)-conjugated goat anti-mouse IgG (Zhongshan Biotechnology, Beijing, China). After further incubation at $37^{\circ} \mathrm{C}$ for $1 \mathrm{~h}$ and thorough washing with PBST, $100 \mu \mathrm{L}$ of $3,3^{\prime}-5,5^{\prime}$-tetramethylbenzidine (TMB) was added as a substrate for HRP. The reaction was stopped after $10 \mathrm{~min}$ with $2 \mathrm{M} \mathrm{H}_{2} \mathrm{SO}_{4}$, and the absorbance was measured at $450 \mathrm{~nm}$ by an ELISA plate reader (Bio-Rad, Hercules, CA). Mannosebinding protein (MBP) was used as negative control.

\section{Western blot analysis}

Virus or prokaryotic expressed fusion proteins were subjected to electrophoresis on $12 \%$ SDS-PAGE after reduction with dithiothreitol (DTT) at $100^{\circ} \mathrm{C}$ for $5 \mathrm{~min}$. The samples were then transferred to a nitrocellulose membrane. Nonspecific antibody binding sites were blocked by immersion of the membrane in $5 \%$ skimmed milk in PBS overnight at $4^{\circ} \mathrm{C}$. The membranes were incubated with MAbs as the primary antibody, and then washed five times with PBST and treated with IRDye 700-conjugated goat anti-mouse secondary antibodies (Licor Biosciences, Lincoln, NE). Blots were visualized by scanning the membranes with a Licor Odyssey infrared image system (Licor Biosciences).

\section{Immunohistochemistry assays}

The $p r M-E$ gene was cloned into the $\mathrm{pCAGGS}^{(14)}$ vector to generate expressing plasmid pCAGGS-WNV-ME and then transfected into BHK21 cells. BHK21 cells expressing prM/E proteins were cultured as monolayers in 24-well cell culture plates. Cells were washed with PBS and fixed with $4 \%$ paraformaldehyde at room temperature for $30 \mathrm{~min}$. Then, cells were washed with PBS and permeabilized with $1 \%$ Triton X100 at $4^{\circ} \mathrm{C}$ for $10 \mathrm{~min}$, after which cells were blocked with $4 \%$ $\mathrm{BSA}$ in PBS at $37^{\circ} \mathrm{C}$ for $30 \mathrm{~min}$. MAbs were added to the cells as the primary antibody and incubated at $37^{\circ} \mathrm{C}$ for $1 \mathrm{~h}$. After washing with gentle shaking in PBS, cells were incubated with HRP-labeled goat anti-mouse IgG (Sigma) diluted 1:2000 in PBS at room temperature for $1 \mathrm{~h}$. After washing three times as described previously, the enzyme reaction was developed with AEC (3-amino-9-ethylcarbazole). Finally, the cells were observed by microscopy (IMT2 Olympus, Tokyo, Japan).

\section{Epitope mapping}

To map the WNV prM protein epitope recognized by the MAb, a set of 20 partially overlapping short peptides (16 amino acids in length, with the exception of the last one,

Table 1. Sequence of Overlapping Peptides DERIVED FROM WNV PRM PROTEIN

\begin{tabular}{|c|c|}
\hline No. of peptide & Peptide sequence \\
\hline prM-1 & ${ }^{1}$ VTLSNFQGKVMMTVNA ${ }^{16}$ \\
\hline prM-2 & ${ }^{8} \mathrm{KVMMTVNATDVTDVIT}^{24}$ \\
\hline prM-3 & ${ }^{17}$ TDVTDVITIPTAAGKN $^{32}$ \\
\hline prM-4 & ${ }^{25}$ IPTAAGKNLCIVRAMD 40 \\
\hline prM-5 & ${ }^{33}$ LCIVRAMDVGYMCDDT $^{48}$ \\
\hline prM-6 & ${ }^{41}$ VGYMCDDTITYECPVL $^{56}$ \\
\hline prM-7 & ${ }^{49}$ ITYECPVLSAGNDPED ${ }^{64}$ \\
\hline prM-8 & ${ }^{57}$ SAGNDPEDIDCWCTKS ${ }^{72}$ \\
\hline prM-9 & ${ }^{65}{ }^{\text {IDCWCTKSAVYVRYGR }}{ }^{80}$ \\
\hline prM-10 & ${ }^{73}$ AVYVRYGRCTKTRHSR ${ }^{88}$ \\
\hline prM-11 & ${ }^{81}$ CTKTRHSRRSRRSLTV $^{96}$ \\
\hline prM-12 & ${ }^{89}$ RSRRSLTVQTHGESTL ${ }^{104}$ \\
\hline prM-13 & ${ }^{97}$ QTHGESTLANKKGAWM ${ }^{112}$ \\
\hline prM-14 & ${ }^{105}$ ANKKGAWMDSTKATRY $^{120}$ \\
\hline prM-15 & ${ }^{113}{ }^{1}$ STKATRYLVKTESWI ${ }^{128}$ \\
\hline prM-16 & ${ }^{121}$ LVKTESWILRNPGYAL $^{136}$ \\
\hline prM-17 & ${ }^{129}$ LRNPGYALVAAVIGWM $^{144}$ \\
\hline prM-18 & ${ }^{137}$ VAAVIGWMLGSNTMQR $^{152}$ \\
\hline prM-19 & 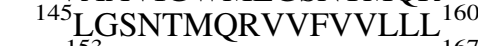 \\
\hline prM-20 & ${ }^{153}$ VVFVVLLLLVAPAYS ${ }^{167}$ \\
\hline
\end{tabular}



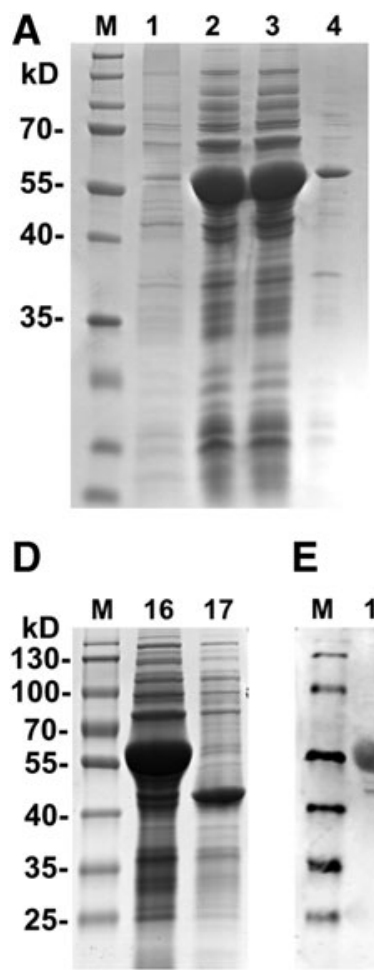
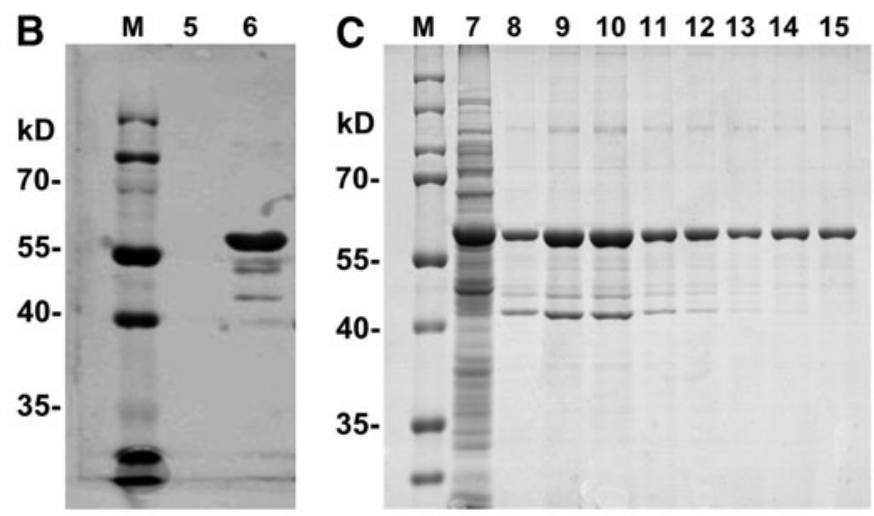

FIG. 1. Expression, purification, and Western blot analysis of WNV prM protein. The expressed MBP fused prM protein was analyzed by SDS-PAGE (A, D) and Western blot with rabbit anti-WNV sera (E) and MBP-specific MAb (B, F). In Western blots $(\mathbf{B}, \mathbf{F})$, the MBP-prM ptotein was probed with a commercial MAb against MBP. In Western blot $(\mathbf{E})$, the MBP-prM and MBP control were probed with rabbit anti-WNV sera. M, protein marker; lanes 1 and 5, uninduced bacterial cell lysates; lanes 2, 6, 16, 18, and 20, induced bacterial cell lysates contain MBP-prM; lane 3, induced bacterial culture supernatant contain MBP-prM; lane 4, induced bacterial precipitate. The MBP-prM fusion protein was purified and analyzed by SDS-PAGE (C). Lane 7, unpurified supernatant; lanes 8-15, fractions of purified MBP-prM protein; lanes 17, 19, and 20, induced bacterial cell lysates contain MBP protein.

which was 15 amino acids) spanning the entire length of the prM protein were designed (Table 1) as described previously. ${ }^{(15)}$ All the fragments were fused with GST and expressed in the pGEX-6p-1 vector. The epitope peptides were screened by indirect ELISA using synthesized peptides as the coating antigen using the method described previously.

\section{Results}

\section{Expression of recombinant prM protein}

To detect the expression of WNV prM in Escherichia coli, the bacterial lysates were analyzed by SDS-PAGE and Western blotting. Results indicated that the recombinant protein was successfully expressed after IPTG induction and was predominantly expressed in a soluble form (Fig. 1A, D). Western blot analysis showed that the recombinant MBPprM protein was recognized by a commercially available MAb against MBP (Fig. 1B, F). SDS-PAGE results showed that the fusion protein was successfully purified using an amylose resin column (Fig. 1C). The recombinant protein MBP-prM could be specifically recognized by rabbit antiWNV sera but MBP protein was not (Fig. 1E).

\section{Preparation of prM protein-specific MAb}

Female BALB/c mice (aged 5 weeks) were immunized with the fusion protein as described in the Materials and

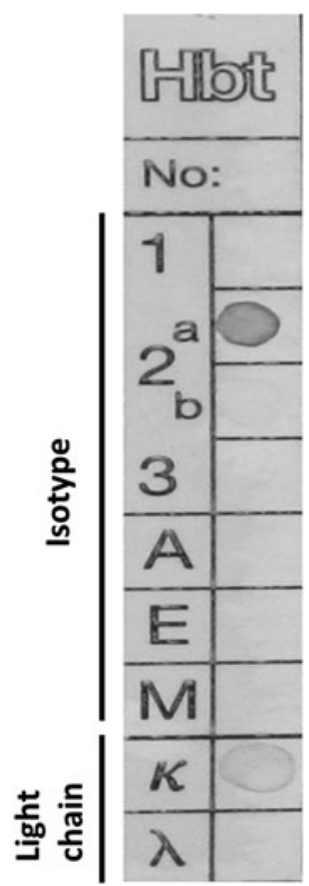

FIG. 2. Isotyping of monoclonal antibody 10F7. The isotype of MAb 10F7 was identified with mouse MAb isotyping kit. Developed dark point shows positive signal. 


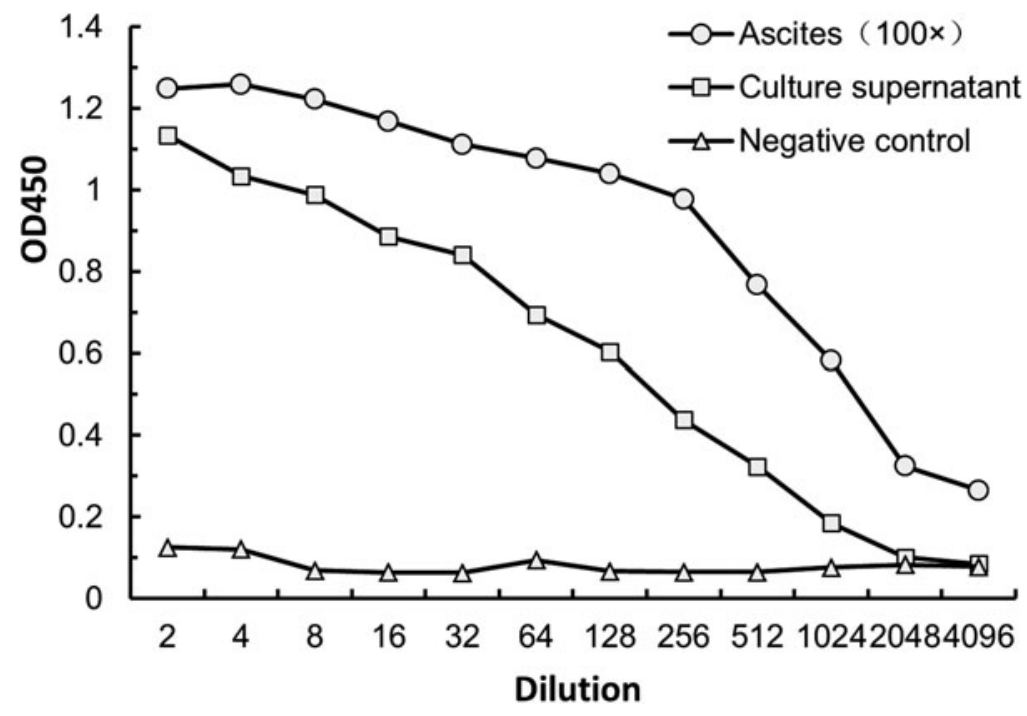

FIG. 3. Titration of MAb 10F7 by ELISA. Culture supernatants and ascites of MAb 10F7 were titered by ELISA. Cell culture medium was used as negative control. Dilution titers of MAb 10F7 in culture supernatants and ascites were 2048 and over 409,600, respectively.

Methods section. After the third immunization, the mouse with the highest antibody titer to the immunogen was selected and a booster immunization was administered prior to cell fusion. After screening with ELISA, one positive clone was obtained. After three rounds of subcloning, one strain showing strong and specific reactivity to the prM protein was selected and designated as 10F7. Isotype investigations revealed that the MAb produced by this clone was identified as the $\operatorname{IgG} 2$ a subtype, and the light chains were $\kappa$ type (Fig. 2).

\section{Biological activity of MAb 10F7}

MAb dilution titers were evaluated by indirect ELISA. The antibody titer of the cell supernatant was 2048, while the titer of ascites fluid exceeded 409,600 (Fig. 3). The specificity and reactivity of the MAbs were further investigated by Western blot analysis using WNV-infected BHK-21 cells and MBPprM protein expressed by $E$. coli ER2523. Western blot analysis suggested that MAb $10 \mathrm{~F} 7$ reacted with WNV-infected cell lysates (Fig. 4A) and MBP-prM (Fig. 4C), demonstrating that the MAbs had good specificity and reactivity, and showed no cross-reactivity with JEV. The ability of specific MAbs to recognize the native prM protein in cells was investigated by immunohistochemistry analysis of BHK-21 cells transfected with the pCAGGS vector containing the $\mathrm{prM}$ gene. A dark red signal was detected, thus confirming that the $\mathrm{MAb}$ recognized the native prM protein (Fig. 5).

\section{Epitope mapping of MAb 10F7}

A set of overlapping synthesized peptides was successfully expressed (Fig. 6A) and used as coating antigens in ELISAs for mapping of the epitope recognized by MAb 10F7. Among these 20 peptides, MAb 10F7 reacted strongly with peptide prM-1 (VTLSNFQGKVMMTVNA) only (Fig. 6B). Western blot analysis showed that MAb 10F7 specifically recognized GST-prM-1 but not GST (Fig. 4D).
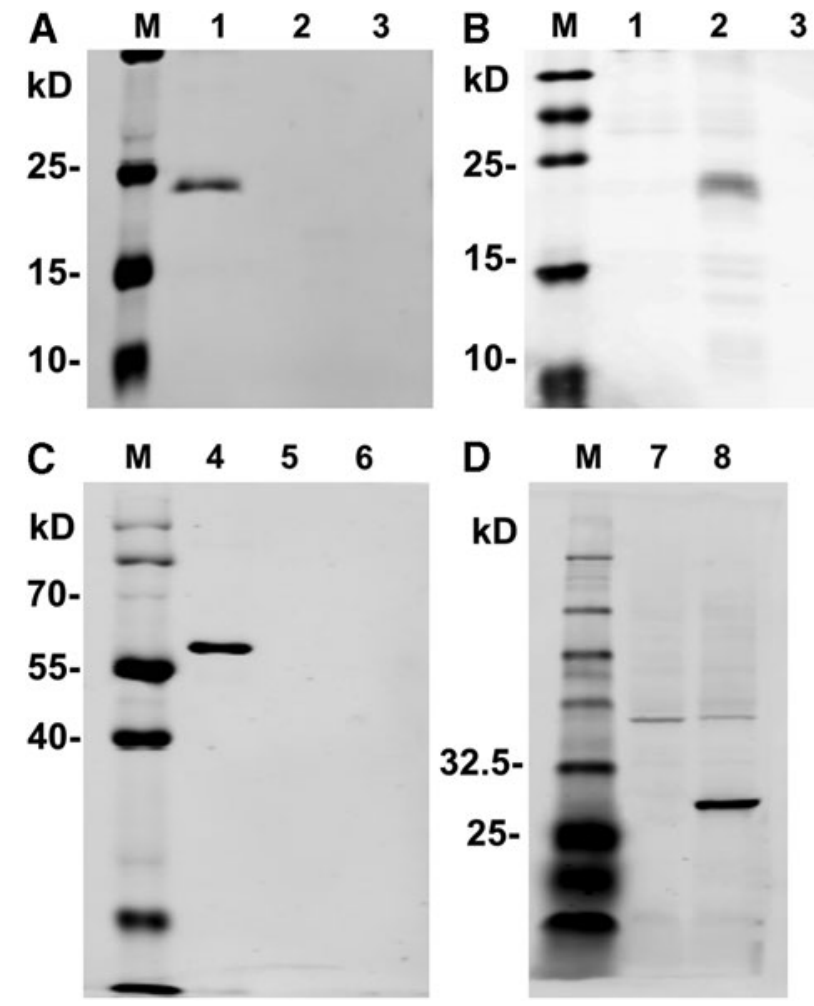

FIG. 4. Western blot analysis of MAb 10F7. Western blots showed that MAb 10F7 specifically recognized prM protein in lysates of cells infected with WNV (A, lane 1) and MBP-prM protein expressed in E. coli (lane 4) and GSTprM-1 (lane 8), while JEV prM specific MAb recognized prM of JEV specifically (B, lane 2). $M$, protein marker; lane 2 , lysates of BHK-21 cells infected with JEV; lane 3, lysates of BHK-21 cells; lane 5, lysates of bacteria expressing MBP; lane 6, uninduced bacterial lysates; lane 7, lysates of bacteria expressing GST. 

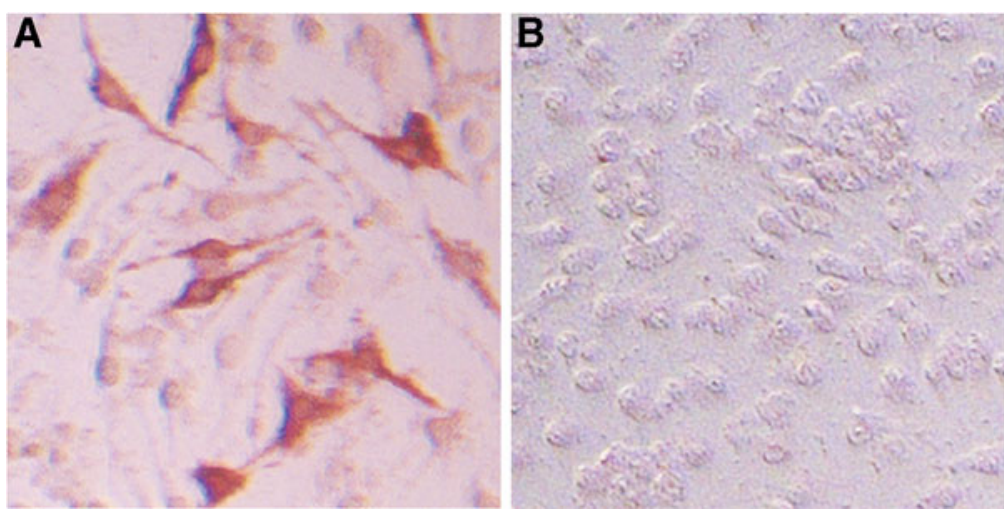

FIG. 5. Micrographs of immunoperoxidase-stained transfected cells. BHK-21 cells transfected with pCAGGS-WNV-ME (A) or untransfected (B) were immunoperoxidase-stained with MAb 10F7 and developed with AEC. Dark red cytoplasmic staining was the result of specific immunoreactivity with prM protein specific MAb $10 \mathrm{~F} 7$.

\section{Discussion}

Following rapid expansion, WNV is now a major threat to human and animal health worldwide. In many areas, WNV co-exists with other flaviviruses, such as JEV in parts of India, St. Louis encephalitis virus (SLEV) on the American continent, and Murray Valley encephalitis virus (MVEV) in Australia. ${ }^{(16)}$ WNV shares some common features with these other flaviviruses and shows cross-reactivity with these viruses in serological assays. These cross-reactive responses could confound the interpretation of serological testing.

It is reported that only mature flavivirus particles are infectious. ${ }^{(17)}$ The cleavage of the prM protein by furin-like protease during virus release from infected cells is necessary for the maturation of flavivirus particles. ${ }^{(18)}$ However, the mechanism of this reaction and the functional role of prM in
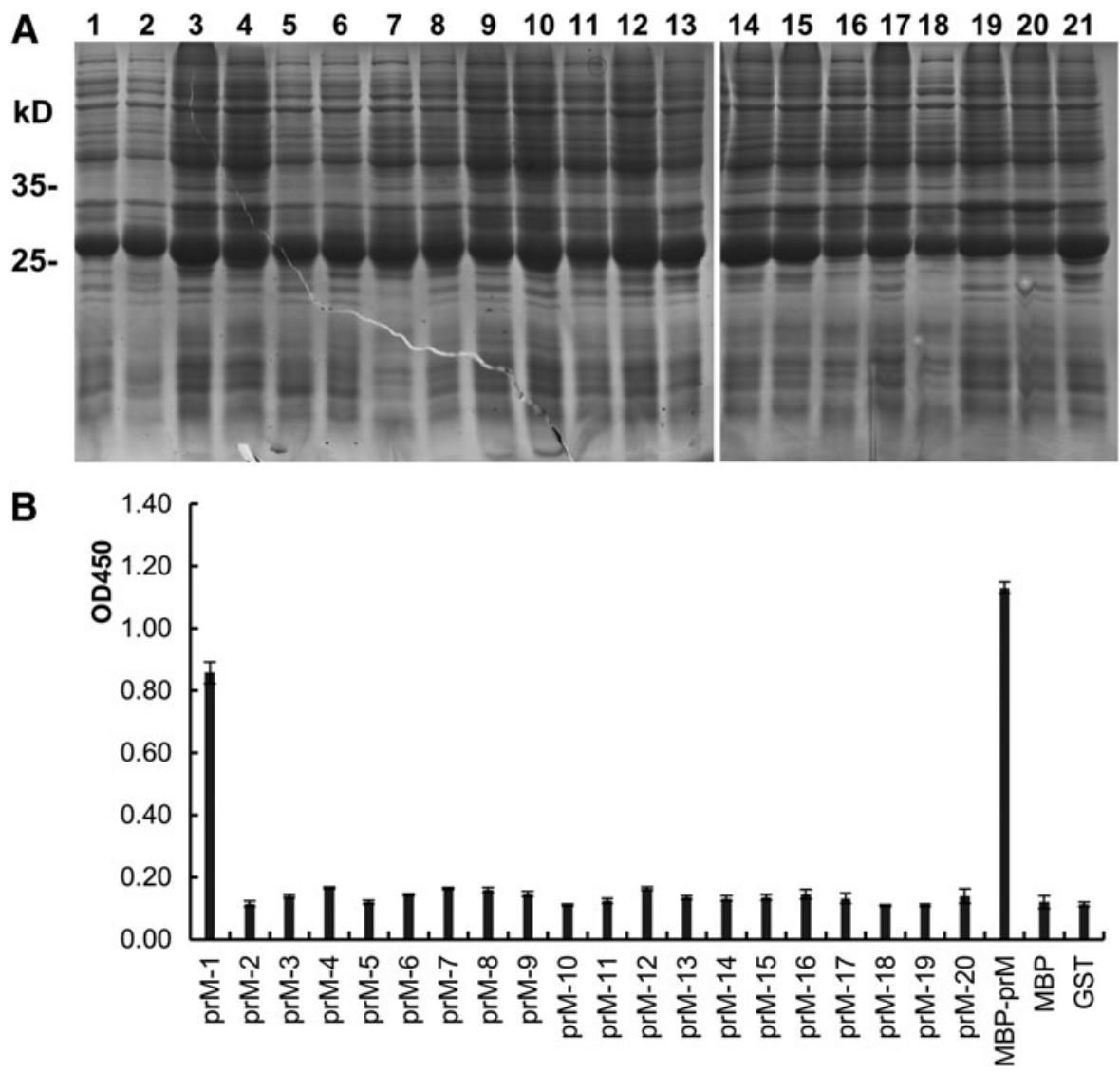

FIG. 6. Peptide expression and epitope mapping. (A) SDS-PAGE analysis of peptides fusion expressed with GST. Lanes 1-20, lysates of bacteria expressing fusion proteins GST-prM-1 to GST-prM-20; lane 21, lysates of bacteria expressing GST. (B) Epitope mapping of MAb 10F7 against WNV prM protein by ELISA. Peptides prM-1 to prM-20 were expressed as GST fusions and screened by indirect ELISA. MBP-prM fusion protein was used as positive control; MBP and GST were used as negative controls, respectively. The values represent mean values of triplicates. 
replication and virus infectivity have not yet been characterized in detail. Previous studies ${ }^{(19,20)}$ indicate that prM has relatively high antigenicity and human sera infected with dengue virus did not show cross-reaction with JEV prM in Western blot analyses and vice versa. ${ }^{(21)}$ These results indicate that prM and antibodies against prM may have potential use for conducting seroepidemiological studies of flavivirus infections in the regions that have prevalence of more than one flavivirus.

In this study, a prokaryotic expression system was used to generate a recombinant protein for use as an antigen to immunize mice. A WNV-prM protein-specific MAb (10F7) was generated using hybridoma technology. Immunohistochemistry assays showed that the $\mathrm{MAb}$ reacted with native prM protein expressed by transfected BHK- 21 cells and epitope mapping showed that the MAb generated specifically recognized the pr-segment of WNV prM protein.

In conclusion, the MAb generated in this study may be useful for investigating the properties and functions of prM and the mechanism of the cleavage reaction in flaviviruses. Furthermore, this MAb represents a potential candidate for development of extensive applications in the diagnosis of WNV infections.

\section{Acknowledgments}

The authors would like to thank Dr. Cheng-Feng Qin for providing the inactive virus antigens of WNV.

\section{Author Disclosure Statement}

The authors have no financial interests to disclose.

\section{References}

1. Garmendia AE, Van Kruiningen HJ, and French RA: The West Nile virus: its recent emergence in North America. Microbes Infect 2001;3(3):223-229.

2. Murgue B, Zeller H, and Deubel V: The ecology and epidemiology of West Nile virus in Africa, Europe and Asia. Curr Top Microbiol Immunol 2002;267:195-221.

3. Tsai TF, Popovici F, Cernescu C, Campbell GL, and Nedelcu NI: West Nile encephalitis epidemic in southeastern Romania. Lancet 1998;352:767-771.

4. Centers for Disease Control and Prevention (CDC). West Nile virus and other arboviral diseases-United States, 2012. MMWR 2013;62:513-517.

5. Mukhopadhyay S, Kim BS, Chipman PR, Rossmann MG, and Kuhn RJ: Structure of West Nile virus. Science 2003;302:248.

6. Ulbert S: West Nile virus: the complex biology of an emerging pathogen. Intervirology 2011;54:171-184.

7. Kuhn RJ, Zhang W, Rossmann MG, Pletnev SV, Corver J, Lenches E, Jones CT, Mukhopadhyay S, Chipman PR, Strauss EG, Baker TS, and Strauss JH: Structure of dengue virus: implications for flavivirus organization, maturation, and fusion. Cell 2002;108:717-725.

8. Yu IM, Holdaway HA, Chipman PR, Kuhn RJ, Rossmann $\mathrm{MG}$, and Chen J: Association of the pr peptides with dengue virus at acidic $\mathrm{pH}$ blocks membrane fusion. J Virol 2009;83:12101-12107.

9. Lorenz IC, Allison SL, Heinz FX, and Helenius A: Folding and dimerization of tick-borne encephalitis virus envelope proteins prM and $\mathrm{E}$ in the endoplasmic reticulum. J Virol 2002;76:5480-5491.

10. Nowak T, Farber PM, Wengler G, and Wengler G: Analyses of the terminal sequences of West Nile virus structural proteins and of the in vitro translation of these proteins allow the proposal of a complete scheme of the proteolytic cleavages involved in their synthesis. Virology 1989;169:365-376.

11. Kaufman BM, Summers PL, Dubois DR, Cohen WH, Gentry MK, Timchak RL, Burke DS, and Eckels KH: Monoclonal antibodies for dengue virus prM glycoprotein protect mice against lethal dengue infection. Am J Trop Med Hyg 1989;41:576-580.

12. Hua RH, and Bu ZG: A monoclonal antibody against PrM/ $M$ protein of Japanese encephalitis virus. Hybridoma 2011;30:451-456.

13. Hua RH, Liu LK, Chen ZS, Li YN, and Bu ZG: Comprehensive mapping antigenic epitopes of NS1 protein of Japanese encephalitis virus with monoclonal antibodies. PLoS One 2013;8:e67553.

14. Jiang Y, Yu K, Zhang H, Zhang P, Li C, Tian G, Li Y, Wang $\mathrm{X}, \mathrm{Ge} \mathrm{J}, \mathrm{Bu} \mathrm{Z}$, and Chen $\mathrm{H}$ : Enhanced protective efficacy of H5 subtype avian influenza DNA vaccine with codon optimized HA gene in a pCAGGS plasmid vector. Antiviral Res 2007;75:234-241.

15. Hua RH, Chen NS, Qin CF, Deng YQ, Ge JY, Wang XJ, Qiao ZJ, Chen WY, Wen ZY, Liu WX, Hu S, and Bu ZG: Identification and characterization of a virus-specific continuous B-cell epitope on the PrM/M protein of Japanese encephalitis virus: potential application in the detection of antibodies to distinguish Japanese encephalitis virus infection from West Nile virus and Dengue virus infections. Virol J 2010;7:249.

16. Mackenzie JS, and Williams DT: The zoonotic flaviviruses of southern, south-eastern and eastern Asia, and Australasia: the potential for emergent viruses. Zoonoses Publ Health 2009;56:338-356.

17. Moesker B, Rodenhuis-Zybert IA, Meijerhof T, Wilschut J, and Smit JM: Characterization of the functional requirements of West Nile virus membrane fusion. J Gen Virol 2010;91:389-393.

18. Stadler K, Allison SL, Schalich J, and Heinz FX: Proteolytic activation of tick-borne encephalitis virus by furin. $\mathrm{J}$ Virol 1997;71:8475-8481.

19. Colpitts TM, Rodenhuis-Zybert I, Moesker B, Wang P, Fikrig E, and Smit JM: prM-antibody renders immature West Nile virus infectious in vivo. J Gen Virol 2011;92:2281-2285.

20. Dejnirattisai W, Jumnainsong A, Onsirisakul N, Fitton P, Vasanawathana S, Limpitikul W, Puttikhunt C, Edwards C, Duangchinda T, Supasa S, Chawansuntati K, Malasit P, Mongkolsapaya J, and Screaton G: Cross-reacting antibodies enhance dengue virus infection in humans. Science 2010;328:745-748.

21. Cardosa MJ, Wang SM, Sum MS, and Tio PH: Antibodies against prM protein distinguish between previous infection with dengue and Japanese encephalitis viruses. BMC Microbiol 2002;2:9.

Address correspondence to: Rong-Hong Hua State Key Laboratory of Veterinary Biotechnology Harbin Veterinary Research Institute Chinese Academy of Agricultural Sciences No.427 Maduan Street Harbin 150001 P.R. China

E-mail: huaronghong@163.com; huaronghong@caas.cn

Received: May 6, 2014 Accepted: September 4, 2014 